\title{
Successful immediate phrenic nerve reconstruction during mediastinal tumor resection
}

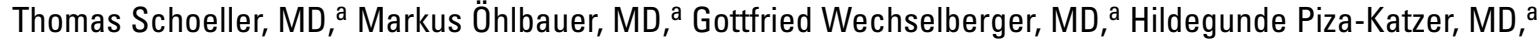 \\ and Raimund Margreiter, MD, Innsbruck, Austria
}

$\mathrm{U}$ nilateral phrenic nerve paralysis is a common lesion in cardiothoracic surgery that usually results in minimal morbidity but may be symptomatic in patients with borderline lung function. ${ }^{1,2}$ In such symptomatic cases plication of the paralyzed hemidiaphragm has been previously suggested to alleviate dyspnea by reducing paradoxic movement

\footnotetext{
From the Clinic for Plastic and Reconstructive Surgery a and Surgery, University of Innsbruck, Innsbruck, Austria.

Received for publication March 22, 2001; accepted for publication April 14, 2001.

Address for reprints: Thomas Schoeller, MD, Department of Plastic and Reconstructive Surgery, University of Innsbruck, Anichstraße 35, A-6020 Innsbruck, Austria (E-mail: Thomas.Schoeller@uibk.ac.at).

J Thorac Cardiovasc Surg 2001;122:1235-7

Copyright $(\odot) 2001$ by The American Association for Thoracic Surgery

$0022-5223 / 2001 \$ 35.00+0 \quad \mathbf{1 2 / 5 4 / 1 1 7 2 7 4}$

doi:10.1067/mtc.2001.117274
}

of the paralyzed hemidiaphragm. ${ }^{3}$ We believed, however, that optimal treatment of diaphragmatic dysfunction caused by tumor infiltration of the phrenic nerve might be immediate microsurgical phrenic nerve reconstruction after curative resection of the tumor.

To our knowledge, this strategy and its feasibility have not been described previously. We here report on our initial experience with this concept.

\section{Clinical Summary}

A 75-year-old woman with a 2-month history of recurrent dyspnea during exercise was referred for diagnostic workup. A chest radiograph demonstrated paralysis of the left hemidiaphragm (Figure 1), most likely caused by a tumor in the anterior mediastinum with infiltration of the left phrenic nerve, as shown by computed tomographic scan (Figure 2).

Through a median sternotomy the tumor was identified and resected en bloc with parts of the affected pericardium, pleura, lung parenchyma, and a patch of the ascending aorta. As was suspected, the left phrenic nerve was infiltrated by the tumor. A $5-\mathrm{cm}$ long piece had to be resected starting $8 \mathrm{~cm}$ from the diaphragm, leaving a $3-\mathrm{cm}$ long distal stump. 


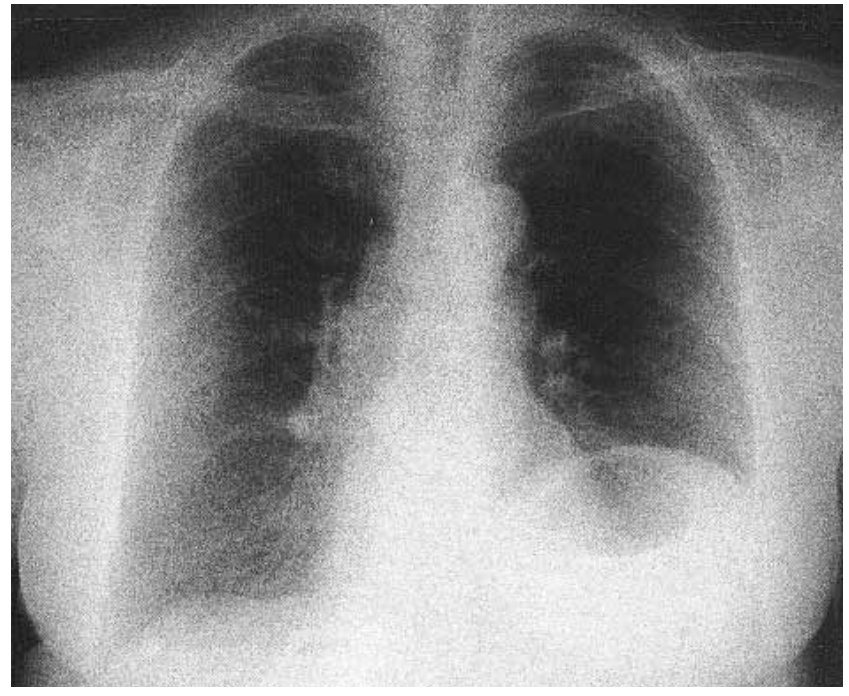

Figure 1. Preoperative chest x-ray film with paralyzed left hemidiaphragm caused by tumor infiltration of the phrenic nerve.

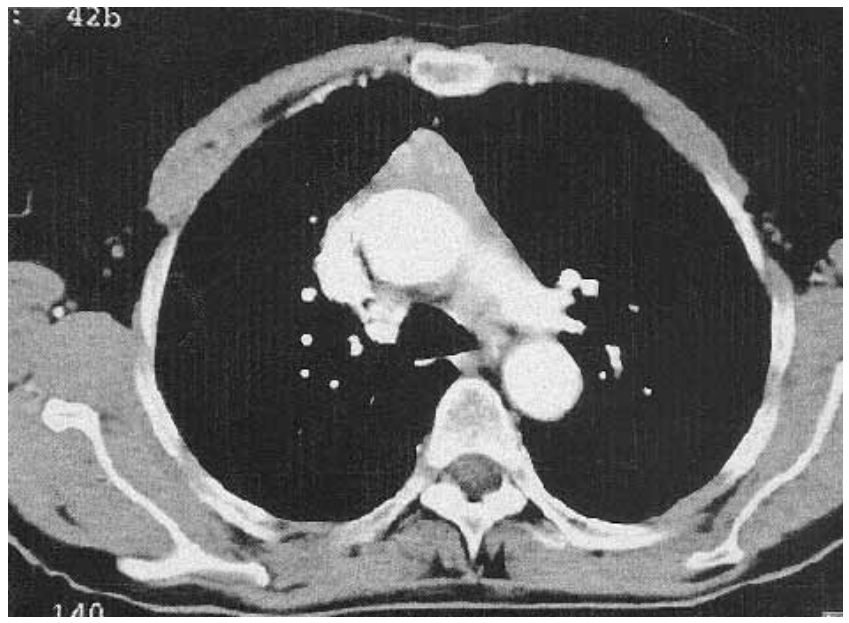

Figure 2. Preoperative computed tomographic scan showing the tumor ventral from the aorta with infiltration of the phrenic nerve.

Because the patient's early signs of dyspnea were more likely caused by phrenic nerve paralysis than by direct tumor compression, immediate phrenic nerve reconstruction with a sural nerve graft from the right calf was considered. End-to-end nerve coaptation was performed with an epineural microsurgical 8-0 Ethilon interrupted suture (Ethicon, Inc, Somerville, NJ) at both graft ends. The nerve suture was secured with $0.5 \mathrm{~mL}$ of topically applied fibrin glue. The sural nerve graft was taken $3 \mathrm{~cm}$ longer than the original phrenic nerve defect to allow extracardiac excursions after partial pericardial resection (Figure 3). Because the patient was already weaned from cardiopulmonary bypass and the beating heart thus did not allow the use of a microscope, reconstruction was performed with magnifying loupes. Histologic and

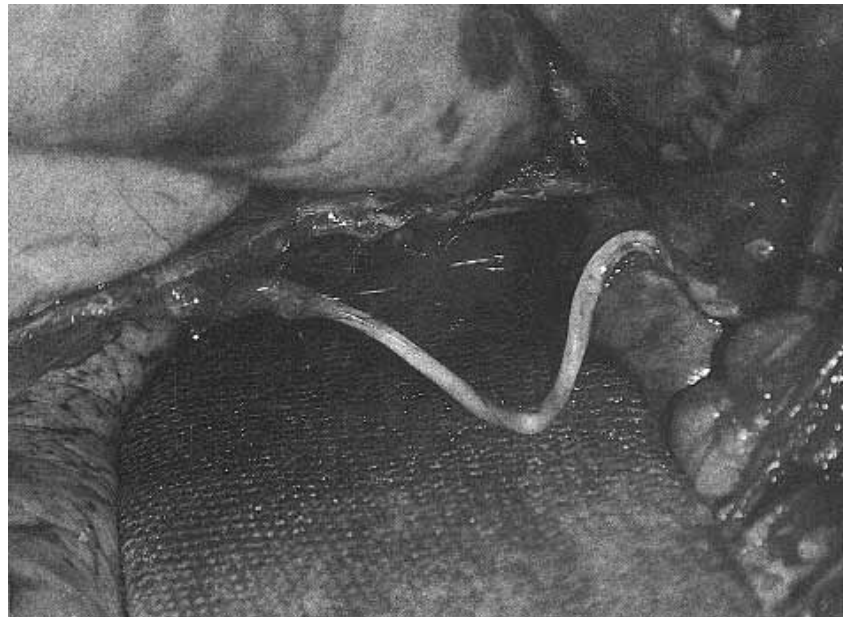

Figure 3. Intraoperative view of the sural nerve graft for phrenic nerve replacement.

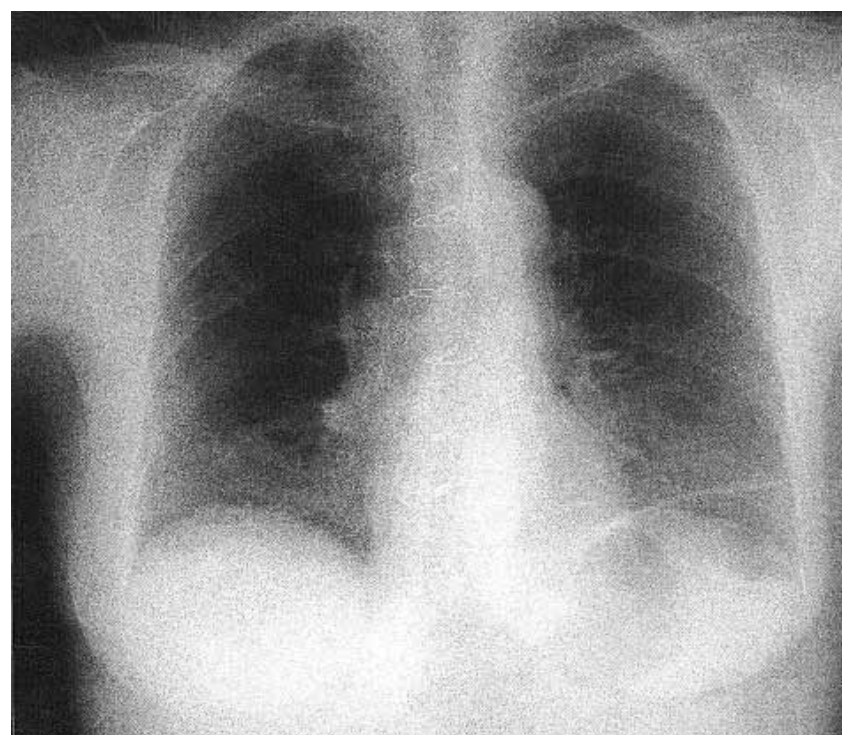

Figure 4. Follow-up chest $x$-ray film after 9 months showing successful reinnervation of the diaphragm.

immunohistochemical examination revealed a malignant thymoma (World Health Organization type C) with clear resection margins. The postoperative course was uneventful.

At 3 months the patient had completely recovered from the operation and, although in excellent physical condition, still had dyspnea. The chest x-ray film still showed an elevated left hemidiaphragm. In contrast, 9 months after reconstruction, the chest radiograph demonstrated both hemidiaphragms at the same level as a sign of successful axonal regeneration of the formerly paralyzed phrenic nerve (Figure 4). Fluoroscopy demonstrated adequate and symmetric motion of both hemidiaphragms. At that time lung function had improved to allow the patient to resume former athletic activities. Comparative surface measurement of nerve con- 
duction velocity on both sides and surface stimulation of the reconstructed phrenic nerve proved that diaphragmatic reinnervation through the nerve graft had been successful.

At the donor site the patient noticed a small numb area at the dorsum of the foot without major discomfort.

\section{Discussion}

The consequences of phrenic nerve injury are variable and depend to a large extent on the individual patient's condition, particularly pulmonary function and age. Pathologic conditions may range from an asymptomatic radiographic abnormality (the phrenic nerve can even be harvested with negligible morbidity as a source for brachial plexus reconstruction in otherwise healthy patients $)^{4}$ to severe pulmonary dysfunction and even mortality in patients with primarily reduced lung function. According to the literature, diaphragmatic plication appears to be the most effective option for patients severely compromised by phrenic nerve injury.

The case presented here demonstrates that irreversible symptomatic hemidiaphragmatic paralysis can be efficiently treated by direct reconstruction with a nerve graft, which is probably less invasive than plication of the diaphragm. This method, however, is recommended only for cases of short-lasting paralysis, because irreversible denervation of a muscle occurs within 1 year after onset of paralysis through motor endplate disintegration. We therefore suggest immediate microsurgical phrenic nerve repair whenever a phrenic nerve lesion is diagnosed either in the context of resection or operative complication under the following conditions: (1) An adequate time frame must be provided to allow com- plete reinnervation considering that a nerve regenerates at a velocity of $1 \mathrm{~mm}$ per day from the proximal nerve coaptation site to the motor endplate in the diaphragm; (2) thoracotomy is performed for other reasons; (3) the patient's general condition must allow an extra operating time of at least 30 minutes for reconstruction without increasing the risk.

This report proves - to our knowledge for the first time-the feasibility of immediate phrenic nerve reconstruction after resection of a malignant tumor. It further shows that the technique of microsurgical repair with sural nerve transfer can be applied safely in phrenic nerve injury with nearly negligible donor-site morbidity. ${ }^{5}$ We believe that in selected cases a nerve graft is an excellent means of reanimating the diaphragm and thus completely restoring the patient's ventilation.

\section{References}

1. Tripp HF, Bolton JW. Phrenic nerve injury following cardiac surgery: a review. J Card Surg. 1998;13:218-23.

2. de Leeuw M, Williams JM, Freedom RM, Williams WG, Shemie SD, McCrindle BW. Impact of diaphragmatic paralysis after cardiothoracic surgery in children. J Thorac Cardiovasc Surg. 1999;118:510-7.

3. Graham DR, Kaplan D, Evans CC, Hind CR, Donnelly RJ. Diaphragmatic plication for unilateral diaphragmatic paralysis: a 10year experience. Ann Thorac Surg. 1990;49:248-51.

4. Gu YD, Ma MK. Use of the phrenic nerve for brachial plexus reconstruction. Clin Orthop. 1996;323:119-21.

5. Ehretsman RL, Novak CB, Mackinnon SE. Subjective recovery of nerve graft donor site. Ann Plast Surg. 1999;43:606-12. 